\title{
Economic Design of Variable Sampling Intervals EWMA Control Charts under Binomial Distribution
}

\author{
XUE Li \\ School of Management Science and Engineering \\ Zhengzhou Institute of Aeronautical Industry Management \\ Zhengzhou, China \\ e-mail: xueli_fys@sina.com
}

\begin{abstract}
Objective-The purpose of this paper is to investigate the economic design of EWMA charts with variable sampling intervals (VSI) under binomial distribution to reduce the cost of control process. MethodsFirst, the economic model of VSI EWMA control chart under binomial distribution is developed, the optimal values of the parameters will be determined such that the expected total cost is minimized. Further, the genetic algorithms will be used to search for the optimal values of the parameters of the VSI EWMA control chart under binomial distribution. Finally, the sensitivity analysis of this model is done to investigate the effect of model parameters on the solution of the economic model. Result-The result shows that the larger the the quality cost per hour while producing in control and the parameters shift of binomial distribution , the smaller the total expected cost and so on. ConclusionsThis research not only provides the ideas and method to other VSI EWMA control charts economic design, but also provides the theoretical basis to reduce the cost of outcontrol process and improves the production efficiency in the actual production.
\end{abstract}

Keywords-variable sampling intervals; binomial distribution; exponential weighted moving average (EWMA) control chart; economic design

\section{INTRODUCTION}

Control charts, which control the production process condition and guarantee the process quality, are the important statistics process control tools. NP control chart which proposed by Shewhart can monitor the number of unqualified products in the production process, and it is established based on binomial distribution (Shewhart, 1924). When the process has small shift, the exponential weighted moving average (EWMA) control charts have the better monitoring efficiency than traditional Shewhart control chart (Roberts, 1959,1966). Saccucci etal firstly proposed variable sampling interval (VSI) EWMA control charts, their results showed that the monitoring efficiency of VSI EWMA control charts excel to the fixed sampling intervals (FSI) EWMA control chart (Saccucci etal, 1992).

Although many author researched the statistical design of VSI EWMA control charts(Reynolds, 2001; Ji, 2006; Xue, 2011), few work was contributed to economic design of VSI EWMA control charts( Chou, 2006). To improve monitoring efficiency and reduce control cost for the number of unqualified products in the production process, the economic design of VSI EWMA control chart under binomial distribution is investigated in this paper.

\section{VSI BINOMIAL EWMA CONTROL CHART}

Assume the quantities of products is infinite and the overall unqualified rate of products is $p$, the sample which the size equal to $n$ is randomly selected from the overall, then the probability which the the nonconforming products equal to $\mathrm{X}$ in the sample is under binomial distribution. The formula is as follows: $P(X=x)=C_{n}^{x} p^{x}(1-p)^{n-x}(0<p<1)$

If EWMA control chart is used to monitor the process, the VSI binomial EWMA statistics is:

$$
Z_{0}=n p, Z_{t}=\lambda X_{t}+(1-\lambda) Z_{t-1}
$$

The control limit UCL $L C L$, central line $C L$ and warning limit $U W L, L W L$ of VSI binomial EWMA is as follows:

$$
\left\{\begin{array}{l}
U C L=n p+k_{1} \sqrt{\frac{\lambda}{2-\lambda} n p(1-p)} \\
U W L=n p+w_{1} \sqrt{\frac{\lambda}{2-\lambda} n p(1-p)} \\
C L=n p \\
L W L=n p-w_{2} \sqrt{\frac{\lambda}{2-\lambda} n p(1-p)} \\
L C L=n p-k_{2} \sqrt{\frac{\lambda}{2-\lambda} n p(1-p)}
\end{array}\right.
$$

Where $\lambda$ is the smoothing coefficient. $k_{1}, k_{2}$ is the upper and lower control limit coefficient, respectively $w_{1}$ and $w_{2}$ are the upper and lower warning limit coefficient respectively, and $0<w_{1}<k_{1}, 0<w_{2}<k_{2}$.

Selecting two sampling intervals $h_{1}$ and $h_{2}\left(h_{1}>h_{2}>0\right)$, if the sample is in located into central domain $[L W L, U W L]$, the longer sample intervals $h_{1}$ will be taken; If the sample is in located into warning domain $[U W L, U C L] \mathrm{Y}[L C L, L W L]$, the shorter intervals $h_{2}$ will be taken; If the sample is exceeded the control limit, the control chart alarms. 


\section{DEVELOPMENT OF ECONOMIC MODEL}

Before the economic model is developed, there are some assumptions. Firstly, the process is assumed in control initially; Secondly, the time which being in control state until out of control is under exponential distribution with parameter $q$; Thirdly, when the process is out of control state, it stays out of control until the process is detected and corrected; Fourthly, the process standard deviation ${ }^{S}$ remains unchanged; Finally, the process will be disturbed by a single assignable cause that causes a fixed shift in the process.

The economic design of VSI EWMA control chart under binomial distribution can be developed by minimizing the expected total cost to determine the optimize of design parameters. The cost model developed by Lorenzen \& Vance (1983) is modified in the present paper to be the objective function for the economic design of the VSI EWMA chart under binomial distribution. The formula of the expected total cost is as follows:

$$
\begin{array}{r}
C=\frac{c_{1} / \theta+c_{2}\left[-\tau+n d+A T S_{1}+\gamma_{1} t_{1}+\gamma_{2} t_{2}\right]+\frac{s g}{A N S S_{0}}+m}{1 / \theta+\left(1-\gamma_{1}\right) \frac{s t_{0}}{A N S S_{0}}-\tau+n d+A T S_{1}+t_{1}+t_{2}} \\
+\left(\frac{a+b n}{h_{0}}\right) \frac{1 / \theta-\tau+n d+A T S_{1}+\gamma_{1} t_{1}+\gamma_{2} t_{2}}{1 / \theta+\left(1-\gamma_{1}\right) \frac{s t_{0}}{A N S S_{0}}-\tau+n d+A T S_{1}+t_{1}+t_{2}}
\end{array}
$$

Where $a$ is fixed cost per sample; cost per unit sampled is denoted as $b ; c_{1}$ and $c_{2}$ represents quality cost per hour while producing in control or out of control, respectively; Denote $d$ as time to sample and chart one item; cost per false alarm is denoted as $g$; Denote $m$ as cost to locate and correct the assignable cause; $s$ represents expected number of samples taken while in control, and it equal to $e^{-\theta h_{0}} /\left(1-e^{-\theta h_{0}}\right) \approx 1 / \theta h_{0} ; \tau$ denote as expected time of occurrence of the assignable cause between two samples. While in control $=\frac{1-\left(1+\theta h_{0}\right) e^{-\theta h_{0}}}{\theta\left(1-e^{-\theta h_{0}}\right)} \approx \frac{h_{0}}{2}-\frac{1}{12} \theta h_{0}{ }^{2} ;$ expected search time when the signal is a false alarm, is denoted as $T_{0} ; T_{1}$ represents expected time to discover the assignable cause; $T_{2}$ represents expected time to correct the process; $\gamma_{1}=\{$, if production continues during searches,

$\gamma_{1}=\{$, if production ceases during searches,

$\gamma_{2}=\{$, if production continues during correction,

0 , if production ceases during correction,

Average sampling interval is denoted as $h_{0}$, and it can be get by

$$
h_{0}=A T S_{0} / A N S S_{0}
$$

Where $A N S S_{0}$ is the average run length when the process is in control, $A T S_{0}$ is the average time to signal when the process is in control. Denote $A T S_{1}$ as average time to signal when the process is out-of-control which mean shifts equal to $\delta$.

The formula of $A T S_{0}$ and $A T S_{1}$ which be obtained by Markov chain method are as follows

$$
: \operatorname{ATS}_{0}=\sum_{i=1}^{2 m+1} q_{k_{0}, i} b_{i}
$$

Define $b\left(b_{1}, b_{2}, \ldots, b_{2 m+1}\right)$, when $Z_{t}$ locate into ith state $E_{i}$, denote the sampling intervals which be selected as $b_{i}(i=1,2, \ldots, 2 m+1)$. When ith state $E_{i}$ locate into $[L W L, U W L]$, the sampling intervals $b_{i}$ equal to $h_{1}$; When the ith state locate into $[U W L, U C L] \mathrm{Y}[L C L, L W L]$, the sampling intervals $b_{i}$ equal to $h_{2}$.

Define

$$
\begin{gathered}
Q=\left[q_{i j}\right]_{(2 m+1) \times(2 m+1)}=(I-P)^{-1} \\
P=\left[p_{i j}\right]_{(2 m+1) \times(2 m+1)}
\end{gathered}
$$

where $\quad p_{i j}=P\left(A<Y_{t}<B\right)$

$$
\begin{gathered}
A=n p-k_{2} \sqrt{\frac{\lambda}{2-\lambda} n p(1-p)}+\frac{[2(j-1)-(1-\lambda)(2 i-1)]}{2(2 m+1) \lambda}\left(k_{1}+k_{2}\right) \sqrt{\frac{\lambda}{2-\lambda} n p(1-p)} \\
B=n p-k_{2} \sqrt{\frac{\lambda}{2-\lambda} n p(1-p)}+\frac{[2 j-(1-\lambda)(2 i-1)]}{2(2 m+1) \lambda}\left(k_{1}+k_{2}\right) \sqrt{\frac{\lambda}{2-\lambda} n p(1-p)}
\end{gathered}
$$

In the same way, we can get:

$$
A T S_{1}=\sum_{i=1}^{2 m+1} q_{k_{0}, i} b_{i}
$$

When the process is out of control, $P=P_{1}$, and

$$
P[Y=y]=\left(\begin{array}{c}
n+y-1 \\
n-1
\end{array}\right) p_{1}^{n}\left(1-p_{1}\right)^{y}, y=0,1,2 \ldots
$$

In the same way, the formula of $\mathrm{ANSS}_{0}$ can be get by Markov chain method:

$\operatorname{ANSS}_{0}=\sum_{j=1}^{2 m+1} q_{k_{0}, i}$

Where the formula of $q_{i j}$ is the same as the $q_{i j}$ in $A T S_{0}$, such as the formula (7)and (8).

The previous discussion shows that the expected total cost is the function of eight parameter $n, h_{1}, h_{2}, k_{1}, k_{2}, \omega_{1}, \omega_{2}, \lambda$. The optimal value of eight parameters can be determined by minimizing the expected total cost through the economic design of VSI EWMA control chart.

\section{AN EXAMPLE}

The genetic algorithm is a kind of macroscopic sense bionic algorithm and it is very effective to solve the large scale optimization problem which has been successfully applied to many fields to solve the optimization problem(Seyed, 2009; Ihsan, 2009).Then the industrial example is given to show how to use genetic algorithm to obtain the optimal solution of economic model of VSI EWMA control chart under binomial distribution.

Suppose that the quality characteristic of a production process is under binomial distribution which the parameter equal to $P$. When the process is in control, $P=P_{0}=0.1 ;$ When the process is out of control, $P=P_{1}=\delta P_{0}=0.15$. The production process can be monitored by VSI EWMA control chart under 
binomial distribution. In this example the cost parameter and model parameter is as follows:

$a=\$ 1, b=\$ 0.3, c_{1}=\$ 15, c_{2}=\$ 80, g=\$ 60, m=\$ 25$, $d=0.1 \mathrm{hr}, t_{0}=0.5 \mathrm{hr}, t_{1}=2 \mathrm{hr}, t_{2}=2 \mathrm{hr}, \theta=0.01$, $\gamma_{1}=\gamma_{2}=1, \delta=1.5$.

For eight parameters $n, h_{1}, h_{2}, k_{1}, k_{2}, W_{1}, W_{2}, \lambda$, the sample size $n$ should be an integer, the other parameters should be continuous values. Using the GA toolbox in matlab 7, we let population size $=20$; crossover probability $=0.8$; mutation rate $=0.1$; the number of generation $=100$; the fitness function= Equation (4). After run the toolbox, we can get the optimal values of $n, h_{1}, h_{2}, k_{1}, k_{2}, W_{1}, W_{2}, \lambda$ as follow:

$n=6, h_{1}=2.328, h_{2}=0.51, k_{1}=1.589, k_{2}=1.226$,

$w_{1}=0.277, w_{2}=1.202, \lambda=0.611, C=26.6989$.

\section{A SENSITIVITY ANALYSIS}

A sensitivity analysis of the economic model of VSI EWMA chart under binomial distribution is conducted using multiple regressions and orthogonal-array experimental design. The effect of model parameters on the solution can be studied based on the sensitivity analysis . The model parameters $\left(a, b, q, c_{1}, c_{2}, d, m, g, t_{1}, t_{2}, d\right)$ are regards as the independent variables, the the expected total cost and eight design parameters $\left(n, h_{1}, h_{2}, k_{1}, k_{2}, w_{1}, w_{2}, \lambda\right)$ are considered as the dependent variables.

Eleven Independent variables and their corresponding two level planning are shown in table I. Then the L16 orthogonal array is employed and there are 16 trials. Genetic algorithm is applied to find the optimal solution of the economic model for each trial. The other model parameters are fixed: $\gamma_{1}=\gamma_{2}=1, t_{0}=1$. The 16 trials selected according the orthogonal Table $L_{16}\left(2^{15}\right)$ are shown in table II , and the results for each trial is also recorded in table III.

TABLE I. Model Parameters AND Their LeVer PANNING

\begin{tabular}{|ccc|}
\hline Model Parameter & Level 1 & Level 2 \\
\hline$c_{1}$ & 4 & 10 \\
$c_{2}$ & 40 & 100 \\
$\theta$ & 0.01 & 0.05 \\
$a$ & 1 & 6 \\
$b$ & 0.1 & 1 \\
$g$ & 30 & 80 \\
$m$ & 20 & 50 \\
$d$ & 0.1 & 0.5 \\
$t_{1}$ & 2 & 6 \\
$t_{2}$ & 2 & 6 \\
$\delta$ & 1.5 & 2 \\
\hline
\end{tabular}

TABLE II. THE 16 TRIALS SELECTED ACCORDING THE ORTHOGONAL TABLE $L_{16}\left(2^{15}\right)$

\begin{tabular}{|c|cccccccccccc|}
\hline Trial & \multicolumn{10}{|l}{ Model Parameter } \\
\cline { 2 - 11 } & \multicolumn{1}{|c}{$c_{1}$} & $c_{2}$ & $\theta$ & $a$ & $b$ & & $d$ & $m$ & $g$ & $t_{1}$ & $t_{2}$ \\
& $\delta$ & & & & & & & & & & \\
\hline 1 & 4 & 40 & 0.01 & 1 & 0.1 & 0.1 & 20 & 30 & 2 & 2 & 1.5 \\
2 & 4 & 40 & 0.01 & 1 & 0.1 & 0.1 & 20 & 80 & 6 & 6 & 2 \\
3 & 4 & 40 & 0.01 & 6 & 1.0 & 0.5 & 50 & 30 & 2 & 2 & 2 \\
4 & 4 & 40 & 0.01 & 6 & 1.0 & 0.5 & 50 & 80 & 6 & 6 & 1.5 \\
5 & 4 & 100 & 0.05 & 1 & 0.1 & 0.5 & 50 & 30 & 6 & 6 & 1.5 \\
6 & 4 & 100 & 0.05 & 1 & 0.1 & 0.5 & 50 & 80 & 2 & 2 & 2 \\
7 & 4 & 100 & 0.05 & 6 & 1.0 & 0.1 & 20 & 30 & 6 & 6 & 2 \\
8 & 4 & 100 & 0.05 & 6 & 1.0 & 0.1 & 20 & 80 & 2 & 2 & 1.5 \\
9 & 10 & 40 & 0.05 & 1 & 1.0 & 0.1 & 50 & 30 & 2 & 6 & 1.5 \\
10 & 10 & 40 & 0.05 & 1 & 1.0 & 0.1 & 50 & 80 & 6 & 2 & 2 \\
11 & 10 & 40 & 0.05 & 6 & 0.1 & 0.5 & 20 & 30 & 2 & 6 & 2 \\
12 & 10 & 40 & 0.05 & 6 & 0.1 & 0.5 & 20 & 80 & 6 & 2 & 1.5 \\
13 & 10 & 100 & 0.01 & 1 & 1.0 & 0.5 & 20 & 30 & 6 & 2 & 1.5 \\
14 & 10 & 100 & 0.01 & 1 & 1.0 & 0.5 & 20 & 80 & 2 & 6 & 2 \\
15 & 10 & 100 & 0.01 & 6 & 0.1 & 0.1 & 50 & 30 & 6 & 2 & 2 \\
16 & 10 & 100 & 0.01 & 6 & 0.1 & 0.1 & 50 & 80 & 2 & 6 & 1.5 \\
\hline
\end{tabular}

TABLE III. RESULTS FOR EACH

\begin{tabular}{|c|c|c|c|c|c|c|c|c|c|}
\hline \multirow[t]{2}{*}{ Trial } & \multicolumn{9}{|c|}{ output } \\
\hline & $n$ & $h_{1}$ & $h_{2}$ & $k_{1}$ & $k_{2}$ & $w_{1}$ & $w_{2}$ & $\lambda$ & \\
\hline 1 & 11 & 2.708 & 80.635 & 1.705 & 1.608 & 0.815 & 0.706 & 60.638 & 310.648 \\
\hline 2 & 14 & 2.627 & 0.137 & 2.824 & 2.714 & 41.005 & 0.961 & 10.286 & 511.594 \\
\hline 3 & 3 & 3.123 & 0.824 & 1.505 & 1.020 & 1.044 & 0.998 & 0.476 & 13.713 \\
\hline 4 & 3 & 3.471 & 0.755 & 1.529 & 1.031 & 1.067 & 1.021 & 0.421 & 18.178 \\
\hline 5 & 3 & 1.222 & 0.168 & 1.502 & 1.038 & 0.676 & 0.418 & 0.472 & 54.707 \\
\hline 6 & 3 & 1.200 & 0.559 & 1.579 & 1.100 & 0.691 & 0.338 & 0.432 & 41.917 \\
\hline 7 & 7 & 2.563 & 0.824 & 1.507 & 1.105 & 1.200 & 1.092 & 0.637 & 56.248 \\
\hline 8 & 6 & 2.047 & 0.842 & 1.562 & 1.028 & 1.018 & 0.990 & 0.732 & 50.557 \\
\hline 9 & 2 & 1.516 & 0.850 & 1.507 & 1.001 & 0.761 & 0.755 & 0.265 & 29.105 \\
\hline 10 & 2 & 1.450 & 0.825 & 1.513 & 1.322 & 1.309 & 1.303 & 0.212 & 28.847 \\
\hline 11 & 6 & 3.179 & 0.600 & 1.526 & 1.091 & 1.359 & 1.066 & 0.670 & 27.980 \\
\hline 12 & 6 & 3.500 & 0.849 & 1.505 & 1.086 & 1.397 & 0.965 & 0.722 & 30.559 \\
\hline 13 & 2 & 1.211 & 0.724 & 1.506 & 1.016 & 1.054 & 0.979 & 0.274 & 28.542 \\
\hline 14 & 2 & 1.265 & 0.016 & 1.579 & 1.100 & 1.101 & 1.076 & 0.310 & 27.808 \\
\hline 15 & 20 & 3.250 & 0.100 & 1.878 & 1.785 & $\begin{array}{lll}5 & 1.318\end{array}$ & 1.289 & 90.505 & 25.441 \\
\hline 16 & 18 & 3.05 & +0.850 & 1.624 & 1.624 & 40.934 & +0.902 & 20.862 & 29.411 \\
\hline
\end{tabular}

For each dependent variable, the statistical software SPSS is used to run the regression analysis to study the effects of model parameters on the solution of economic model of VSI EWMA chart under binomial distribution. From the output of SPSS with dependent variables, we can get the model parameters which significantly impact on them, respectively. The results are as follow:

The larger the fixed cost per sample ${ }^{a}$, quality cost per hour while producing in control ${ }^{c_{1}}$, quality cost per hour while producing out of control $c_{2}$, expected time to discover the assignable cause ${ }^{t_{1}}$ and the parameters shift of binomial distribution $\delta$, the larger the sample size $n$; The larger the occurrences frequency of the assignable cause $\theta$, cost per unit sampled $b$ and time to sample and chart one item $d$, the smaller the sample size $n$.

(2) An increase in the fixed cost per sample $a$ lengthens the long sampling interval ${ }^{h}$; An increase in the occurrences frequency of the assignable cause $\theta$, cost per 
unit sampled $b$ and quality cost per hour while producing out of control $c_{2}$ shortens the long sampling interval $h_{1}$. Meanwhile, we find that no model parameter significantly affects the short sampling interval $h_{2}$.

(3) The cost per unit sampled $b$, time to sample and chart one item $d$ and the occurrences frequency of the assignable cause $\theta$ significantly affect the lower control limit coefficient $k_{2}$. And the larger the three model parameters, the smaller the lower control limit coefficient $k_{2}$. Meanwhile, we find that no model parameter significantly affects the upper control limit coefficient $k_{1}$.

(4) The larger quality cost per hour while producing in control ${ }^{c_{1}}$, the fixed cost per sample ${ }^{a}$,expected time to discover the assignable cause ${ }^{t_{1}}$ and the parameters shift of binomial distribution $\delta$, the larger the warning limit coefficient $w_{1}$; The larger quality cost per hour while producing out of control $c_{2}$, cost to locate and correct the assignable cause $m$ and expected time to correct the process $t_{2}$, the smaller the warning limit coefficient $w_{1}$.

(5) An increase in the quality cost per hour while producing in control $c_{1}$, the fixed cost per sample $a$,expected time to discover the assignable cause $t_{1}$, the parameters shift of binomial distribution $\delta$ and cost per unit sampled $b$ lenghth lower warning limit coefficient ${ }^{w_{2}}$; An increase in the occurrences frequency of the assignable cause $\theta$ and time to sample and chart one item $d$ shortens lower warning limit coefficient $w_{2}$.

(6) The larger the fixed cost per sample $a$, the larger the exponential weight constant $\lambda$; The larger the cost per unit sampled $b$,cost to locate and correct the assignable cause $m$, expected time to discover the assignable cause $^{t_{1}}$ and the parameters shift of binomial distribution $\delta$, the smaller the smoothing coefficient $\lambda$.

\section{CONCLUSIONS}

The economic design of VSI EWMA chart under binomial distribution is developed in this paper. The values of the eight test parameters of the control chart can be determined through minimizing the expected total cost. The genetic algorithm is used to obtain the optimal solution of economic model and an illustrative example is provided. The effect of model parameters on the solution of the economic model can be obtained from a sensitivity analysis.

\section{ACKNOWLEDGMENT}

This research was supported by the National Science Foundation of China under grant U1204702, Aeronautic Science Foundation of China under grant 2013ZG55024, and Program for Science \& Technology research projects sponsored plan of the education department of Henan Province under grant 14A630018.

\section{REFERENCES}

[1] Chou, C.H. and Chen, C.H. (2006), "Economic design of EWMA charts with variable sampling intervals", Quality \& Quantity, Vol.40, pp.879-896

[2] Ihsan K. (2009), "A genetic algorithm approach to determine the sample size for control charts with variables and attributes", Expert Systems with Applications, Vol 36, No. 5, pp.8719-8734.

[3] Ji, M.M. and Sun, H. (2007), "An EWMA standard deviation control chart with variable sampling intervals", Mathematics in practice and theory, Vol.37, No.12, pp.90-96.

[4] Lorenzen, T.J. and Vance, L.C. (1986), "The economic design of control charts: a unified approach", Technometrics, Vol. 28, pp. 310.

[5] Reynolds, M.R., Arnold, J.C. (2001), "EWMA control charts with variable sample sizes and variable sampling intervals", IIE Transactions, Vol. 33, pp. 511-530.

[6] Roberts, S.W. (1959), "Control chart tests based on geometric moving averages", Technometrics, Vol.1, pp. 239-250.

[7] Roberts, S W.(1966), "A comparison of some control chart procedures". Technometrics, Vol.8, No.3, pp.411-430.

[8] Saccucci, M.S. and Lucas, J.M. (1990), “Average run lengths for exponentially weighted moving average control schemes using the Markov chain approach", Journal of Quality Technology, Vol.22, pp. 154-162.

[9] Shewhart W. A.(1926), "Quality control charts", Bell Systems Technical Journal, Vol.2, pp.593-560.

[10] Seyed T., Mohammad J.E., Mahdi M. (2009), "Economic and economic-statistical designs of MEWMA control charts-a hybrid Taguchi loss, Markov chain, and genetic algorithm approach” Int J Adv Manuf Technol, DOI 10.1007/s00170-009-2288-0.

[11] Xue L.(2011), "EWMA control chart with variable sampling intervals under geometric distribution", Statistics and decision,Vol. 19 , pp.43-45. 